SUPPLEMENTARY MATERIAL

for the

communication

entitled

\title{
An Anomeric Control on Remote Stereochemistry in the Synthesis of Spiroketals.
}

authored by

Sunil K. Ghosh, Richard P. Hsung*, and Jia Liu

Department of Chemistry, University of Minnesota, Minneapolis, MN 55455 


\section{General Procedure for Metallation and Reaction with Acrolein.}

To a solution of 3,4-dihydro- $2 H$-pyran $(1.50 \mathrm{~g}, 26.7 \mathrm{mmol})$ in THF $(50 \mathrm{~mL})$ at $-78{ }^{0} \mathrm{C}$ was added $t$-BuLi $(11.5$ $\mathrm{mL}$ of a $1.7 \mathrm{M}$ in pentane, $19.6 \mathrm{mmol}, 1.2$ equiv). After stirring at $0{ }^{0} \mathrm{C}$ for $45 \mathrm{~min}$, it was cooled to $-78{ }^{0} \mathrm{C}$, and a solution of acrolein $(1.50 \mathrm{~g}, 26.7 \mathrm{mmol})$ in THF $(10 \mathrm{~mL})$ was added and the resulting mixture was stirred for an additional hour at this temperature. The reaction mixture was quenched by sat aq $\mathrm{NH}_{4} \mathrm{Cl}(30 \mathrm{~mL})$ at $-78{ }^{0} \mathrm{C}$, allowed to warm up to rt, and extracted with ether $(3 \times 50 \mathrm{~mL})$. The combined organic layers were washed with sat aq $\mathrm{NaCl}(50 \mathrm{~mL})$, dried over $\mathrm{Na}_{2} \mathrm{SO}_{4}$, and concentrated under reduced pressure. The crude mixture was filtered through a small bed of silica gel column (20\% EtOAc in hexanes) to give the alcohol as colorless oil.

To a solution of the alcohol prepared above in $\mathrm{CH}_{2} \mathrm{Cl}_{2}(50 \mathrm{~mL})$ were added pyridine $(15 \mathrm{~mL})$ and $\mathrm{Ac} c_{2} \mathrm{O}(2.0$ $\mathrm{mL}$ ) at $\mathrm{rt}$ and the resulting mixture was stirred for $24 \mathrm{~h}$. After a standard aqueous work up, the acetate was purified using silica gel flash column chromatography (Isocratic eluent: 15\% EtOAc in hexanes) to give $1.45 \mathrm{~g}$ $(90 \%)$ of product as colorless oil.

\section{General Procedure for the Preparation of the Precursors for Pauson-Khand Reactions.}

To a solution of the acetate prepared above $(1.00 \mathrm{~g}, 5.49 \mathrm{mmol})$ and propargyl alcohol $(0.92 \mathrm{~g}, 16.4 \mathrm{mmol}, 3$ equiv) in anhy $\mathrm{CH}_{2} \mathrm{Cl}_{2}(50 \mathrm{~mL})$ was added PPTS (pyridinium $p$-toluenesulfonate) (125.0 mg, $0.50 \mathrm{mmol}, 10$ mol\%) at $\mathrm{rt}$ and the resulting mixture was refluxed for $12 \mathrm{~h}$. The solvent was removed under reduced pressure and the crude product was purified by flash chromatography (Gradient eluent: 2-5\% EtOAc in hexanes) to afford $0.70 \mathrm{~g}(70 \%)$ of 7 with the anti $[\mathbf{7 a}]$ to $s y n[7 \mathrm{~s}]$ ratio being $1.1: 1$ (separable by column chromatography).

\section{General Procedure for Pauson-Khand Cycloaddition Reactions.}

A mixture of ketal 7a $(50.0 \mathrm{mg}, 0.21 \mathrm{mmol})$ and $\mathrm{Co}_{2}(\mathrm{CO})_{8}(85.0 \mathrm{mg}, 0.25 \mathrm{mmol}, 1.2$ equiv) in toluene $(70 \mathrm{~mL})$ was stirred at room temperature for $1 \mathrm{~h}$. To this mixture was added trimethylamine $N$-oxide $(19.0 \mathrm{mg}, 0.25$ mmol, 1.2 equiv) and the resulting mixture was refluxed for $1 \mathrm{~h}$. The solvent was removed under reduced pressure and the crude product was purified by flash chromatography (Gradient eluent: 15-40\% EtOAc in hexanes) to afford $0.50 \mathrm{~g}(91 \%)$ of cycloadduct 8 . 


\section{Precursors for Pauson-Khand Cycloaddition Reactions.}

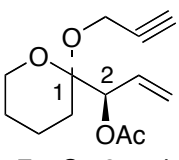

7a: C1-2 anti

$\mathrm{R}_{f}=0.29$ [10\% EtOAc in hexanes]; ${ }^{1} \mathrm{H}$ NMR $\left(500 \mathrm{MHz}, \mathrm{CDCl}_{3}\right) \delta 1.48-1.60(\mathrm{~m}, 4 \mathrm{H}), 1.72-1.81(\mathrm{~m}, 2 \mathrm{H}), 2.11$ $(\mathrm{s}, 3 \mathrm{H}), 2.37-2.38(\mathrm{~m}, 1 \mathrm{H}), 3.64-3.71(\mathrm{~m}, 2 \mathrm{H}), 4.16-4.25(\mathrm{~m}, 2 \mathrm{H}), 5.20-5.24(\mathrm{~m}, 2 \mathrm{H}), 5.34-5.35(\mathrm{~m}, 1 \mathrm{H}), 5.84-$ $5.90(\mathrm{~m}, 1 \mathrm{H}) ;{ }^{13} \mathrm{C} \mathrm{NMR}\left(125 \mathrm{MHz}, \mathrm{CDCl}_{3}\right) \delta 17.9,21.0,24.8,28.3,48.7,62.1,73.3,73.8,80.4,99.0,117.3$, 132.7, 169.6; IR (neat) $\mathrm{cm}^{-1} 3290 \mathrm{~m}, 2947 \mathrm{~s}, 2878 \mathrm{~m}, 1746 \mathrm{~s}$; mass spectrum (LCMS) for $\mathrm{C}_{13} \mathrm{H}_{18} \mathrm{O}_{4}$ : m/e (relative intensity): 183 (20) (M-O-propargyl) ${ }^{+}, 123$ (100).

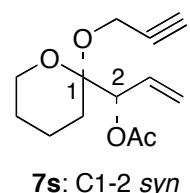

$\mathrm{R}_{f}=0.25\left[10 \%\right.$ EtOAc in hexanes]; ${ }^{1} \mathrm{H}$ NMR $\left(500 \mathrm{MHz}, \mathrm{CDCl}_{3}\right) \delta 1.49-1.80(\mathrm{~m}, 6 \mathrm{H}), 2.11(\mathrm{~s}, 3 \mathrm{H}), 2.41(\mathrm{t}, J=$ $2.5 \mathrm{~Hz}, 1 \mathrm{H}), 3.60-3.71(\mathrm{~m}, 2 \mathrm{H}), 4.21-4.30(\mathrm{~m}, 2 \mathrm{H}), 5.21-5.27(\mathrm{~m}, 2 \mathrm{H}), 5.40-5.41(\mathrm{~m}, 1 \mathrm{H}), 5.86-5.93(\mathrm{~m}, 1 \mathrm{H})$; ${ }^{13} \mathrm{C}$ NMR $\left(125 \mathrm{MHz}, \mathrm{CDCl}_{3}\right) \delta$ 18.0, 21.1, 24.5, 27.9, 48.8, 62.4, 73.7, 74.0, 80.3, 99.3, 117.8, 131.8, 169.8; IR (neat) $\mathrm{cm}^{-1} 3293 \mathrm{~m}, 2945 \mathrm{~s}, 2875 \mathrm{~m}, 1741 \mathrm{~s}$; mass spectrum (LCMS) for $\mathrm{C}_{13} \mathrm{H}_{18} \mathrm{O}_{4}$ : m/e (relative intensity): 183 (20) (M-O-propargyl) $)^{+}, 123(100)$.

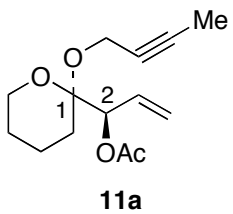

$\mathrm{R}_{f}=0.37\left[10 \%\right.$ EtOAc in hexanes]; ${ }^{1} \mathrm{H}$ NMR $\left(500 \mathrm{MHz}, \mathrm{CDCl}_{3}\right) \delta 1.49-1.60(\mathrm{~m}, 4 \mathrm{H}), 1.72-1.86(\mathrm{~m}, 2 \mathrm{H}), 1.86$ $(\mathrm{s}, 3 \mathrm{H}), 2.11(\mathrm{~s}, 3 \mathrm{H}), 3.64-3.70(\mathrm{~m}, 2 \mathrm{H}), 4.16-4.18(\mathrm{~m}, 2 \mathrm{H}), 5.19-5.24(\mathrm{~m}, 2 \mathrm{H}), 5.36-5.37(\mathrm{~m}, 1 \mathrm{H}), 5.85-5.92(\mathrm{~m}$, $1 \mathrm{H}) ;{ }^{13} \mathrm{C} \mathrm{NMR}\left(125 \mathrm{MHz}, \mathrm{CDCl}_{3}\right) \delta$ 3.7, 17.9, 21.0, 24.8, 28.2, 49.1, 61.9, 73.7, 75.5, 81.3, 98.9, 117.0, 132.9, 169.6; IR (neat) $\mathrm{cm}^{-1} 2947 \mathrm{~s}, 2876 \mathrm{~m}, 1746 \mathrm{~s}$; mass spectrum (LCMS) for $\mathrm{C}_{14} \mathrm{H}_{20} \mathrm{O}_{4}$ : m/e (relative intensity): 183 (26) (M-O-propargyl) ${ }^{+}, 123(100)$.

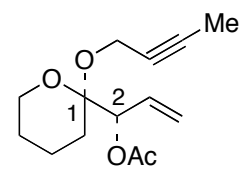

$11 \mathrm{~s}$

$\mathrm{R}_{f}=0.32\left[10 \%\right.$ EtOAc in hexanes]; ${ }^{1} \mathrm{H}$ NMR $\left(500 \mathrm{MHz}, \mathrm{CDCl}_{3}\right) \delta 1.50-1.85(\mathrm{~m}, 6 \mathrm{H}), 1.86(\mathrm{~s}, 3 \mathrm{H}), 2.12(\mathrm{~s}, 3 \mathrm{H})$, 3.62-3.72 (m, 2H), 4.22-4.24 (m, 2H), 5.23-5.28 (m, 2H), 5.44-5.45 (m, 1H), 5.89-5.96 (m, $1 \mathrm{H})$; ${ }^{13} \mathrm{C}$ NMR (125 $\left.\mathrm{MHz}, \mathrm{CDCl}_{3}\right) \delta$ 3.7, 18.0, 21.2, 24.6, 27.9, 49.3, 62.3, 74.4, 75.5, 81.7, 99.1, 117.6, 131.9, 169.8; IR (neat) $\mathrm{cm}^{-1}$ 
2945s, 2878m, 1741s; mass spectrum (LCMS) for $\mathrm{C}_{14} \mathrm{H}_{20} \mathrm{O}_{4}: \mathrm{m} / \mathrm{e}$ (relative intensity): 183 (40) (M-Opropargyl) $)^{+}, 123(100)$.

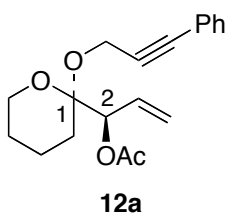

$\mathrm{R}_{f}=0.30$ [10\% EtOAc in hexanes]; ${ }^{1} \mathrm{H}$ NMR $\left(500 \mathrm{MHz}, \mathrm{CDCl}_{3}\right) \delta 1.53-1.67(\mathrm{~m}, 4 \mathrm{H}), 1.77-1.86(\mathrm{~m}, 2 \mathrm{H}), 2.10$ (s, 3H), 3.72-3.78 (m, 2H), 4.41-4.49 (m, 2H), 5.22-5.27 (m, 2H), 5.41-5.42 (m, 1H), 5.89-5.95 (m, 1H), 7.287.30 (m, 3H), 7.41-7.46 (m, 2H); ${ }^{13} \mathrm{C}$ NMR (125 MHz, $\left.\mathrm{CDCl}_{3}\right) \delta$ 18.0, 21.0, 24.9, 28.3, 49.4, 62.1, 73.9, 85.0, 85.7, 99.1, 117.2, 122.8, 128.2, 128.3, 131.8, 132.9, 169.7; IR (neat) $\mathrm{cm}^{-1} 2944 \mathrm{~s}, 2872 \mathrm{~s}, 1746 \mathrm{~s}$; mass spectrum (LCMS) for $\mathrm{C}_{19} \mathrm{H}_{22} \mathrm{O}_{4}: \mathrm{m} / \mathrm{e}$ (relative intensity): 255 (5) (M-OAc) ${ }^{+}, 183$ (20), 123 (100).

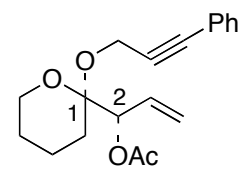

$12 s$

$\mathrm{R}_{f}=0.20$ [10\% EtOAc in hexanes]; ${ }^{1} \mathrm{H}$ NMR $\left(500 \mathrm{MHz}, \mathrm{CDCl}_{3}\right) \delta 1.52-1.72(\mathrm{~m}, 5 \mathrm{H}), 1.78-1.85(\mathrm{~m}, 1 \mathrm{H}), 2.13$ $(\mathrm{s}, 3 \mathrm{H}), 3.69-3.77(\mathrm{~m}, 2 \mathrm{H}), 4.51(\mathrm{~s}, 2 \mathrm{H}), 5.25-5.31(\mathrm{~m}, 2 \mathrm{H}), 5.53-5.54(\mathrm{~m}, 1 \mathrm{H})$, 5.96-6.03 (m, 1H), 7.28-7.30 (m, 3H), 7.44-7.45 (m, 2H); ${ }^{13} \mathrm{C}$ NMR (125 MHz, $\left.\mathrm{CDCl}_{3}\right) \delta 18.1,21.2,24.6,28.0,49.6,62.4,74.5,85.4,85.8,99.4$, 117.7, 122.7, 128.2, 128.3, 131.7, 132.0, 169.8; IR (neat) $\mathrm{cm}^{-1} 2944 \mathrm{~s}, 2872 \mathrm{~s}, 1746 \mathrm{~s}$; mass spectrum (LCMS) for $\mathrm{C}_{19} \mathrm{H}_{22} \mathrm{O}_{4}: \mathrm{m} / \mathrm{e}$ (relative intensity): 255 (5) (M-OAc) ${ }^{+}, 183$ (20), 123 (100).

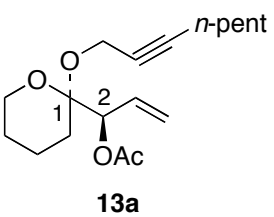

$\mathrm{R}_{f}=0.56\left[10 \%\right.$ EtOAc in hexanes]; ${ }^{1} \mathrm{H} \mathrm{NMR}\left(500 \mathrm{MHz}, \mathrm{CDCl}_{3}\right) \delta 0.87(\mathrm{t}, J=7.0 \mathrm{~Hz}, 3 \mathrm{H}), 1.26-1.35(\mathrm{~m}, 4 \mathrm{H})$, 1.45-1.58 (m, 6H), 1.72-1.79 (m, 2H), $2.10(\mathrm{~s}, 3 \mathrm{H}), 2.16-2.19(\mathrm{~m}, 2 \mathrm{H}), 3.67-3.69(\mathrm{~m}, 2 \mathrm{H}), 4.12-4.22(\mathrm{~m}, 2 \mathrm{H})$, 5.17-5.22 (m, 2H), 5.34-5.35 (m, 1H), 5.84-5.87 (m, 1H); ${ }^{13} \mathrm{C}$ NMR (125 MHz, $\left.\mathrm{CDCl}_{3}\right) \delta$ 13.9, 18.0, 18.8, 21.0, 22.2, 24.9, 28.2, 28.3, 31.0, 49.2, 61.9, 73.8, 76.3, 85.9, 98.8, 117.0, 132.9, 169.6; IR (neat) cm ${ }^{-1} 2936 \mathrm{~s}, 2874 \mathrm{~m}$, 1749s; mass spectrum (LCMS) for $\mathrm{C}_{18} \mathrm{H}_{28} \mathrm{O}_{4}$ : m/e (relative intensity): 249 (5) (M-OAc) ${ }^{+}, 183$ (22), 123 (100).

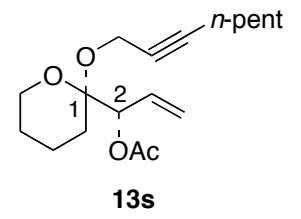

$\mathrm{R}_{f}=0.46\left[10 \%\right.$ EtOAc in hexanes]; ${ }^{1} \mathrm{H}$ NMR $\left(500 \mathrm{MHz}, \mathrm{CDCl}_{3}\right) \delta 0.88(\mathrm{t}, J=7.0 \mathrm{~Hz}, 3 \mathrm{H}), 1.28-1.36(\mathrm{~m}, 4 \mathrm{H})$, 1.47-1.68 (m, 7H), 1.77-1.82 (m, 1H), $2.11(\mathrm{~s}, 3 \mathrm{H}), 2.17-2.21(\mathrm{~m}, 2 \mathrm{H}), 3.65-3.70(\mathrm{~m}, 2 \mathrm{H}), 4.24-4.26(\mathrm{~m}, 2 \mathrm{H})$, 
5.21-5.26 (m, 2H), 5.44-5.46 (m, 1H), 5.90-5.97 (m, 1H); ${ }^{13} \mathrm{C}$ NMR (125 MHz, $\left.\mathrm{CDCl}_{3}\right) \delta$ 13.9, 18.0, 18.8, 21.2, 22.2, 24.7, 27.9, 28.2, 31.0, 49.4, 62.3, 74.4, 76.4, 86.3, 99.2, 117.4, 132.0, 169.8; IR (neat) cm ${ }^{-1} 2934 \mathrm{~s}, 2875 \mathrm{~m}$, $1745 \mathrm{~s}$; mass spectrum (LCMS) for $\mathrm{C}_{18} \mathrm{H}_{28} \mathrm{O}_{4}$ : m/e (relative intensity): 249 (5) (M-OAc) ${ }^{+}, 183$ (22), 123 (100).

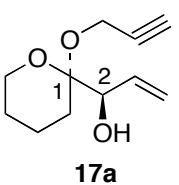

$\mathrm{R}_{f}=0.43\left[20 \%\right.$ EtOAc in hexanes]; ${ }^{1} \mathrm{H}$ NMR $\left(500 \mathrm{MHz}, \mathrm{CDCl}_{3}\right) \delta 1.37-1.52(\mathrm{~m}, 4 \mathrm{H}), 1.62-1.67$ (m, $\left.2 \mathrm{H}\right), 2.43$ (s, $1 \mathrm{H}), 2.56-2.58(\mathrm{~m}, 1 \mathrm{H}), 3.52-3.55(\mathrm{~m}, 1 \mathrm{H}), 3.62-3.64(\mathrm{~m}, 1 \mathrm{H}), 4.12-4.16(\mathrm{~m}, 2 \mathrm{H}), 4.25-4.29(\mathrm{~m}, 1 \mathrm{H}), 5.14-$ $5.18(\mathrm{~m}, 1 \mathrm{H}), 5.31-5.36(\mathrm{~m}, 1 \mathrm{H}), 5.80-5.89(\mathrm{~m}, 1 \mathrm{H}) ;{ }^{13} \mathrm{C} \mathrm{NMR}\left(125 \mathrm{MHz}, \mathrm{CDCl}_{3}\right) \delta$ 17.9, 24.9, 27.8, 48.2, 62.0, 73.7, 73.8, 81.0, 100.0, 116.3, 135.2; IR (neat) $\mathrm{cm}^{-1} 3480 \mathrm{~s}, 3295 \mathrm{~s}, 2948 \mathrm{~s}, 2872 \mathrm{~s}$; mass spectrum (LCMS) for $\mathrm{C}_{11} \mathrm{H}_{16} \mathrm{O}_{3}: \mathrm{m} / \mathrm{e}$ (relative intensity): 139 (18) (M-O-propargyl) ${ }^{+}, 123$ (100).

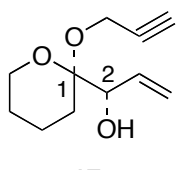

$\mathrm{R}_{f}=0.33$ [20\% EtOAc in hexanes]; ${ }^{1} \mathrm{H}$ NMR (500 MHz, $\left.\mathrm{CDCl}_{3}\right) \delta 1.49-1.80(\mathrm{~m}, 6 \mathrm{H}), 2.39-2.42(\mathrm{~m}, 1 \mathrm{H}), 3.65-$ $3.76(\mathrm{~m}, 2 \mathrm{H}), 4.21-4.24(\mathrm{~m}, 1 \mathrm{H}), 4.24(\mathrm{~s}, 2 \mathrm{H}), 5.21-5.24(\mathrm{~m}, 1 \mathrm{H}), 5.38-5.42(\mathrm{~m}, 1 \mathrm{H}), 5.89-5.95(\mathrm{~m}, 1 \mathrm{H}) ;{ }^{13} \mathrm{C}$ $\operatorname{NMR}\left(125 \mathrm{MHz}, \mathrm{CDCl}_{3}\right) \delta 17.9,24.7,26.8,48.6,62.5,72.9,73.6,80.5,100.4,116.6,134.1$; IR (neat) $\mathrm{cm}^{-1}$ $3482 \mathrm{~s}, 3297 \mathrm{~s}, 2946 \mathrm{~s}, 2875 \mathrm{~s}$; mass spectrum (LCMS) for $\mathrm{C}_{11} \mathrm{H}_{16} \mathrm{O}_{3}: \mathrm{m} / \mathrm{e}$ (relative intensity): 139 (18) (M-Opropargyl) $)^{+}, 123(100)$.

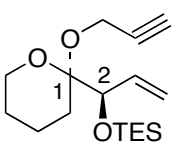

$18 \mathrm{a}$

$\mathrm{R}_{f}=0.57\left[10 \%\right.$ EtOAc in hexanes]; ${ }^{1} \mathrm{H} \mathrm{NMR}\left(500 \mathrm{MHz}, \mathrm{CDCl}_{3}\right) \delta 0.60(\mathrm{q}, J=8.0 \mathrm{~Hz}, 6 \mathrm{H}), 0.95(\mathrm{t}, J=8.0 \mathrm{~Hz}$, $9 \mathrm{H}), 1.35(\mathrm{ddd}, J=4.5,13.5$ and $18.5 \mathrm{~Hz}, 1 \mathrm{H}), 1.47-1.58(\mathrm{~m}, 3 \mathrm{H}), 1.70-1.82(\mathrm{~m}, 2 \mathrm{H}), 2.37(\mathrm{t}, J=2.5 \mathrm{~Hz}, 1 \mathrm{H})$, 3.66-3.69 (m, 2H), $4.05(\mathrm{~d}, J=6.5 \mathrm{~Hz}, 1 \mathrm{H}), 4.27-4.35(\mathrm{~m}, 2 \mathrm{H}), 5.16-5.18(\mathrm{~m}, 1 \mathrm{H}), 5.23-5.27(\mathrm{~m}, 1 \mathrm{H}), 5.87-5.93$ $(\mathrm{m}, 1 \mathrm{H}) ;{ }^{13} \mathrm{C}$ NMR $\left(125 \mathrm{MHz}, \mathrm{CDCl}_{3}\right) \delta$ 5.0, 6.8, 18.3, 25.0, 29.1, 49.3, 61.8, 72.7, 77.0, 81.2, 99.5, 116.4, 137.5; IR (neat) $\mathrm{cm}^{-1} 3312 \mathrm{w}, 2952 \mathrm{~s}, 2880 \mathrm{~s}$; mass spectrum (LCMS) for $\mathrm{C}_{17} \mathrm{H}_{30} \mathrm{O}_{3} \mathrm{Si}$ : m/e (relative intensity): 255 (5) (M-O-propargyl) ${ }^{+}, 156$ (100).

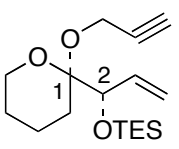

$18 s$ 
$\mathrm{R}_{f}=0.54$ [10\% EtOAc in hexanes]; ${ }^{1} \mathrm{H}$ NMR $\left(500 \mathrm{MHz}, \mathrm{CDCl}_{3}\right) \delta$ 0.59-0.65 (m, 6H), 0.94-0.96 (m, 9H), $1.45-$ $1.76(\mathrm{~m}, 6 \mathrm{H}), 2.39-2.40(\mathrm{~m}, 1 \mathrm{H}), 3.62-3.71(\mathrm{~m}, 2 \mathrm{H}), 4.16-4.21(\mathrm{~m}, 2 \mathrm{H}), 4.25-4.29(\mathrm{~m}, 1 \mathrm{H}), 5.15-5.17(\mathrm{~m}, 1 \mathrm{H})$, 5.32-5.35 (m, 1H), 5.96-6.02 (m, 1H); ${ }^{13} \mathrm{C} \mathrm{NMR}\left(125 \mathrm{MHz}, \mathrm{CDCl}_{3}\right) \delta$ 4.9, 6.8, 18.1, 24.7, 26.2, 48.3, 62.1, 73.2, 74.5, 80.8, 100.8, 115.6, 136.5; IR (neat) $\mathrm{cm}^{-1} 3311 \mathrm{w}, 2952 \mathrm{~s}, 2880 \mathrm{~s}$; mass spectrum (LCMS) for $\mathrm{C}_{17} \mathrm{H}_{30} \mathrm{O}_{3} \mathrm{Si}: \mathrm{m} / \mathrm{e}$ (relative intensity): 255 (5)(M-O-propargyl) ${ }^{+}, 156$ (100).

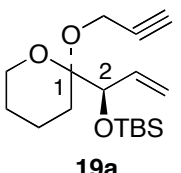

$\mathrm{R}_{f}=0.28$ [5\% EtOAc in hexanes]; ${ }^{1} \mathrm{H}$ NMR (500 MHz, $\left.\mathrm{CDCl}_{3}\right) \delta 0.02(\mathrm{~s}, 3 \mathrm{H}), 0.07$ (s, 3H), 0.90 (s, $\left.9 \mathrm{H}\right), 1.36$ $(\mathrm{ddd}, J=4.5,13.0$ and 18.0 Hz, 1H), 1.49-1.60 (m, 3H), 1.75-1.82 (m, 2H), $2.38(\mathrm{t}, J=2.5 \mathrm{~Hz}, 1 \mathrm{H}), 3.66-3.68$ $(\mathrm{m}, 2 \mathrm{H}), 4.02-4.03(\mathrm{~m}, 1 \mathrm{H}), 4.29-4.30(\mathrm{~m}, 2 \mathrm{H}), 5.15-5.18(\mathrm{~m}, 1 \mathrm{H}), 5.22-5.26(\mathrm{~m}, 1 \mathrm{H}), 5.85-5.95(\mathrm{~m}, 1 \mathrm{H}) ;{ }^{13} \mathrm{C}$ $\operatorname{NMR}\left(125 \mathrm{MHz}, \mathrm{CDCl}_{3}\right) \delta-4.9,-4.4,18.2,18.3,24.9,25.7,29.2,49.3,61.8,72.7,77.2,81.2,99.4,116.5$, 137.4; IR (neat) $\mathrm{cm}^{-1} 3315 \mathrm{~m}, 2950 \mathrm{~s}, 2855 \mathrm{~s}$; mass spectrum (LCMS) for $\mathrm{C}_{17} \mathrm{H}_{30} \mathrm{O}_{3} \mathrm{Si}$ : m/e (relative intensity): 255 (10) (M-O-propargyl) ${ }^{+}, 156(100)$.

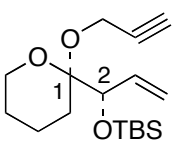

19s

$\mathrm{R}_{f}=0.26\left[5 \%\right.$ EtOAc in hexanes]; ${ }^{1} \mathrm{H}$ NMR $\left(500 \mathrm{MHz}, \mathrm{CDCl}_{3}\right) \delta 0.03(\mathrm{~s}, 3 \mathrm{H}), 0.07(\mathrm{~s}, 3 \mathrm{H}), 0.90(\mathrm{~s}, 9 \mathrm{H}), 1.42-$ $1.74(\mathrm{~m}, 6 \mathrm{H}), 2.38(\mathrm{t}, J=2.5 \mathrm{~Hz}, 1 \mathrm{H}), 3.64-3.68(\mathrm{~m}, 2 \mathrm{H}), 4.14-4.28(\mathrm{~m}, 3 \mathrm{H}), 5.13-5.16(\mathrm{~m}, 1 \mathrm{H}), 5.31-5.36(\mathrm{~m}$, 1H), 5.95-6.14 (m, 1H); ${ }^{13} \mathrm{C}$ NMR (125 MHz, $\left.\mathrm{CDCl}_{3}\right) \delta-4.9,-4.6,18.0,18.3,24.7,25.8,26.1,48.2,62.0,73.2$, 74.3, 80.8, 100.8, 115.5, 136.3; IR (neat) $\mathrm{cm}^{-1} 3314 \mathrm{~m}, 2953 \mathrm{~s}, 2859 \mathrm{~s}$; mass spectrum (LCMS) for $\mathrm{C}_{17} \mathrm{H}_{30} \mathrm{O}_{3} \mathrm{Si}_{\text {: }}$ $\mathrm{m} / \mathrm{e}$ (relative intensity): 255 (12) (M-O-propargyl) ${ }^{+}, 156(100)$.

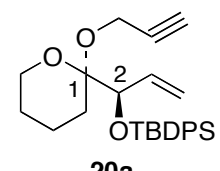

$\mathrm{R}_{f}=0.56\left[10 \%\right.$ EtOAc in hexanes]; ${ }^{1} \mathrm{H}$ NMR $\left(500 \mathrm{MHz}, \mathrm{CDCl}_{3}\right) \delta 1.07(\mathrm{~s}, 9 \mathrm{H}), 1.36-1.60(\mathrm{~m}, 4 \mathrm{H}), 1.82-1.85$ $(\mathrm{m}, 2 \mathrm{H}), 2.35(\mathrm{t}, J=2.5 \mathrm{~Hz}, 1 \mathrm{H}), 3.62-3.67(\mathrm{~m}, 2 \mathrm{H}), 3.94(\mathrm{~d}, J=7.0 \mathrm{~Hz}, 1 \mathrm{H}), 4.04(\mathrm{dd}, J=2.5 \mathrm{and} 15.0 \mathrm{~Hz}$, $1 \mathrm{H}), 4.21(\mathrm{dd}, J=2.5$ and $15.0 \mathrm{~Hz}, 1 \mathrm{H}), 4.77(\mathrm{~d}, J=17.0 \mathrm{~Hz}, 1 \mathrm{H}), 4.95(\mathrm{~d}, J=15.5 \mathrm{~Hz}, 1 \mathrm{H}), 5.79-5.86(\mathrm{~m}, 1 \mathrm{H})$, 7.31-7.41 (m, 6H), 7.61-7.63 (m, 2H), 7.73-7.75 (m, 2H); ${ }^{13} \mathrm{C}$ NMR (125 MHz, $\left.\mathrm{CDCl}_{3}\right) \delta 18.3,19.4,24.9,27.0$, 29.1, 49.0, 61.7, 72.7, 77.9, 81.2, 99.6, 117.9, 127.1, 127.3, 129.4, 129.5, 133.4, 134.1, 136.1, 136.2, 136.5; IR (neat) $\mathrm{cm}^{-1} 3307 \mathrm{~s}, 3072 \mathrm{~m}, 2936 \mathrm{~s}, 2860 \mathrm{~s}$; mass spectrum (LCMS) for $\mathrm{C}_{27} \mathrm{H}_{34} \mathrm{O}_{3} \mathrm{Si}: \mathrm{m} / \mathrm{e}$ (relative intensity): 379 (100) (M-O-propargyl) $)^{+}, 280(50)$. 


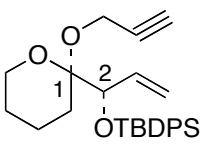

20s

$\mathrm{R}_{f}=0.25$ [5\% EtOAc in hexanes]; ${ }^{1} \mathrm{H}$ NMR $\left(500 \mathrm{MHz}, \mathrm{CDCl}_{3}\right) \delta 1.09(\mathrm{~s}, 9 \mathrm{H}), 1.41-1.76(\mathrm{~m}, 5 \mathrm{H}), 1.98(\mathrm{ddd}, J$ $=5.0,13.0$ and $18.0 \mathrm{~Hz}, 1 \mathrm{H}), 2.29(\mathrm{t}, J=2.5 \mathrm{~Hz}, 1 \mathrm{H}), 3.48-3.58(\mathrm{~m}, 2 \mathrm{H}), 3.83(\mathrm{dd}, J=3.0$ and $15.0 \mathrm{~Hz}, 1 \mathrm{H})$, $4.00(\mathrm{dd}, J=3.0$ and $15.0 \mathrm{~Hz}, 1 \mathrm{H}), 4.17(\mathrm{~d}, J=6.5 \mathrm{~Hz}, 1 \mathrm{H}), 5.03-5.09(\mathrm{~m}, 2 \mathrm{H}), 5.83-5.88(\mathrm{~m}, 1 \mathrm{H}), 7.32-7.40$ $(\mathrm{m}, 6 \mathrm{H}), 7.65-7.73(\mathrm{~m}, 4 \mathrm{H}) ;{ }^{13} \mathrm{C} \mathrm{NMR}\left(125 \mathrm{MHz}, \mathrm{CDCl}_{3}\right) \delta$ 18.1, 19.5, 24.6, 26.5, 27.0, 47.9, 61.8, 73.0, 75.6, $80.6,100.7,116.9,127.0,127.1,129.3,129.4,134.0,134.2,135.7,136.2,136.3$; IR (neat) cm ${ }^{-1} 3308 \mathrm{~s}, 3075 \mathrm{~m}$, 2934s, 2859s; mass spectrum (LCMS) for $\mathrm{C}_{27} \mathrm{H}_{34} \mathrm{O}_{3} \mathrm{Si}: \mathrm{m} / \mathrm{e}$ (relative intensity): 379 (100) (M-O-propargyl) ${ }^{+}$, 280 (60).

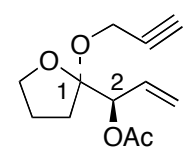

28a

$\mathrm{R}_{f}=0.28\left[10 \%\right.$ EtOAc in hexanes]; ${ }^{1} \mathrm{H}$ NMR $\left(500 \mathrm{MHz}, \mathrm{CDCl}_{3}\right) \delta 1.80-1.88(\mathrm{~m}, 1 \mathrm{H}), 1.96-1.97(\mathrm{~m}, 2 \mathrm{H}), 2.03-$ $2.10(\mathrm{~m}, 1 \mathrm{H}), 2.13(\mathrm{~s}, 3 \mathrm{H}), 2.36(\mathrm{t}, J=2.5 \mathrm{~Hz}, 1 \mathrm{H}), 3.92(\mathrm{q}, J=7.0 \mathrm{~Hz}, 1 \mathrm{H}), 4.10-4.06(\mathrm{~m}, 1 \mathrm{H}), 4.16-4.19$ (m, $1 \mathrm{H}), 4.24-4.28(\mathrm{~m}, 1 \mathrm{H}), 5.24-5.31(\mathrm{~m}, 2 \mathrm{H}), 5.58-5.59(\mathrm{~m}, 1 \mathrm{H}), 5.81-5.89(\mathrm{~m}, 1 \mathrm{H}) ;{ }^{13} \mathrm{C} \mathrm{NMR}\left(125 \mathrm{MHz} \mathrm{CDCl}_{3}\right)$

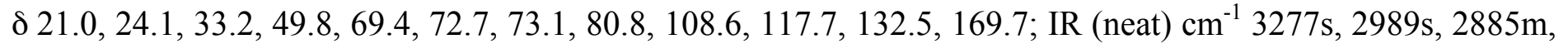
$1748 \mathrm{~s}$; mass spectrum (LCMS) for $\mathrm{C}_{12} \mathrm{H}_{16} \mathrm{O}_{4}$ : m/e (relative intensity): 169 (100) (M-O-propargyl) ${ }^{+}$.

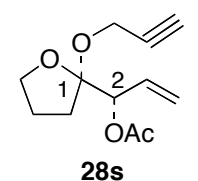

$\mathrm{R}_{f}=0.24$ [10\% EtOAc in hexanes]; ${ }^{1} \mathrm{H}$ NMR (500 MHz, $\left.\mathrm{CDCl}_{3}\right) \delta$ 1.86-1.94 (m, 2H), 2.01-2.08 (m, $\left.2 \mathrm{H}\right), 2.10$ $(\mathrm{s}, 3 \mathrm{H}), 2.40(\mathrm{t}, J=2.5 \mathrm{~Hz}, 1 \mathrm{H}), 3.89-3.93(\mathrm{~m}, 1 \mathrm{H}), 4.02-4.06(\mathrm{~m}, 1 \mathrm{H}), 4.21-4.29(\mathrm{~m}, 2 \mathrm{H}), 5.24-5.34(\mathrm{~m}, 2 \mathrm{H})$, 5.61-5.62 (m, 1H), 5.91-5.98 (m, 1H); ${ }^{13} \mathrm{C}$ NMR (125 MHz, $\left.\mathrm{CDCl}_{3}\right) \delta$ 21.0, 24.1, 32.5, 49.8, 69.8, 72.6, 73.2, 80.6, 109.1, 117.7, 132.6, 169.6; IR (neat) $\mathrm{cm}^{-1} 3275 \mathrm{~s}, 2990 \mathrm{~s}, 2882 \mathrm{~m}, 1745 \mathrm{~s}$; mass spectrum (LCMS) for $\mathrm{C}_{12} \mathrm{H}_{16} \mathrm{O}_{4}: \mathrm{m} / \mathrm{e}$ (relative intensity): 169 (100) (M-O-propargyl) ${ }^{+}$

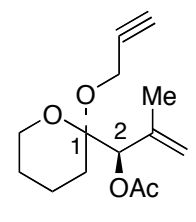

$31 \mathrm{a}$ 
$\mathrm{R}_{f}=0.42\left[10 \%\right.$ EtOAc in hexanes]; ${ }^{1} \mathrm{H}$ NMR $\left(500 \mathrm{MHz}, \mathrm{CDCl}_{3}\right) \delta 1.52(\mathrm{~m}, 2 \mathrm{H}), 1.63(\mathrm{~m}, 2 \mathrm{H}), 1.75(\mathrm{~m}, 2 \mathrm{H})$, $1.84(\mathrm{~s}, 3 \mathrm{H}), 2.10(\mathrm{~s}, 3 \mathrm{H}), 2.38(\mathrm{t}, J=2.5 \mathrm{~Hz}, 1 \mathrm{H}), 3.69(\mathrm{~m}, 2 \mathrm{H}), 4.20(\mathrm{dd}, J=2.3,15.0 \mathrm{~Hz}, 1 \mathrm{H}), 4.27$ (dd, $J=$ 2.3, $15.0 \mathrm{~Hz}, 1 \mathrm{H}), 4.98(\mathrm{~s}, 1 \mathrm{H}), 4.99(\mathrm{~s}, 1 \mathrm{H}), 5.14(\mathrm{~s}, 1 \mathrm{H}) ;{ }^{13} \mathrm{C} \mathrm{NMR}\left(75 \mathrm{MHz}, \mathrm{CDCl}_{3}\right) \delta$ 18.1, 21.2, 21.4, 24.9, 28.1, 48.7, 62.0, 73.3, 76.3, 80.5, 99.6, 113.9, 141.6, 169.5; IR (film) $\mathrm{cm}^{-1} 3277 \mathrm{w}, 2949 \mathrm{~m}, 1745 \mathrm{~s}$; mass spectrum for $\mathrm{C}_{14} \mathrm{H}_{20} \mathrm{O}_{4}$ (ESI): m/e 275.1.

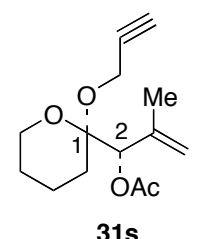

$\mathrm{R}_{f}=0.35$ [10\% EtOAc in hexanes]; ${ }^{1} \mathrm{H}$ NMR $\left(300 \mathrm{MHz}, \mathrm{CDCl}_{3}\right) \delta 1.55(\mathrm{~m}, 2 \mathrm{H}), 1.68(\mathrm{~m}, 3 \mathrm{H}), 1.77(\mathrm{~m}, 1 \mathrm{H})$, $1.87(\mathrm{~s}, 3 \mathrm{H}), 2.13(\mathrm{~s}, 3 \mathrm{H}), 2.43(\mathrm{t}, J=2.4 \mathrm{~Hz}, 1 \mathrm{H}), 3.75(\mathrm{~m}, 2 \mathrm{H}), 4.30(\mathrm{dd}, J=2.4,15.3 \mathrm{~Hz}, 1 \mathrm{H}), 4.37(\mathrm{dd}, J=$ 2.4, $15.3 \mathrm{~Hz}, 1 \mathrm{H}), 5.02$ (t, $J=1.5 \mathrm{~Hz}, 1 \mathrm{H}), 5.06(\mathrm{~s}, 1 \mathrm{H}), 5.23(\mathrm{~s}, 1 \mathrm{H}) ;{ }^{13} \mathrm{C} \mathrm{NMR}\left(75 \mathrm{MHz}, \mathrm{CDCl}_{3}\right) \delta$ 18.3, 20.6, 21.4, 24.7, 29.0, 49.5, 62.6, 73.5, 77.1, 80.5, 99.5, 115.4, 140.4, 169.8; IR (film) $\mathrm{cm}^{-1} 3275 \mathrm{w}, 2946 \mathrm{~m}, 1744 \mathrm{~s}$; mass spectrum for $\mathrm{C}_{14} \mathrm{H}_{20} \mathrm{O}_{4}$ (ESI): m/e 275.1 .

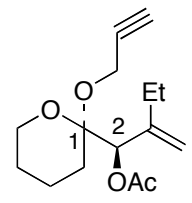

\section{$32 a$}

$\mathrm{R}_{f}=0.26$ [5\% EtOAc in hexanes]; ${ }^{1} \mathrm{H}$ NMR (500 MHz, $\left.\mathrm{CDCl}_{3}\right) \delta 1.03(\mathrm{t}, J=8.0 \mathrm{~Hz}, 3 \mathrm{H}), 1.47-1.81(\mathrm{~m}, 6 \mathrm{H})$, $2.08(\mathrm{~s}, 3 \mathrm{H}), 2.15-2.21(\mathrm{~m}, 2 \mathrm{H}), 2.37(\mathrm{t}, J=2.5 \mathrm{~Hz}, 1 \mathrm{H}), 3.62-3.69(\mathrm{~m}, 2 \mathrm{H}), 4.16-4.27(\mathrm{~m}, 2 \mathrm{H}), 4.99(\mathrm{~s}, 1 \mathrm{H})$, $5.03(\mathrm{~s}, 1 \mathrm{H}), 5.17(\mathrm{~s}, 1 \mathrm{H}) ;{ }^{13} \mathrm{C} \mathrm{NMR}\left(125 \mathrm{MHz}, \mathrm{CDCl}_{3}\right) \delta 12.1,18.0,21.2,24.8,26.8,27.8,48.5,61.9,73.1$, $75.4,80.5,99.8,111.3,147.5,169.5$; IR (neat) $\mathrm{cm}^{-1} 3286 \mathrm{~s}, 2967 \mathrm{~s}, 2946 \mathrm{~s}, 2878 \mathrm{~s}, 1744 \mathrm{~s}$; mass spectrum (LCMS) for $\mathrm{C}_{15} \mathrm{H}_{22} \mathrm{O}_{4}: \mathrm{m} / \mathrm{e}$ (relative intensity): 211 (20) (M-O-propargyl) ${ }^{+}, 151$ (100).

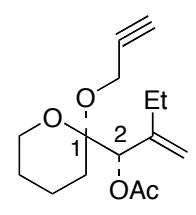

$32 s$

$\mathrm{R}_{f}=0.23$ [5\% EtOAc in hexanes]; ${ }^{1} \mathrm{H}$ NMR $\left(500 \mathrm{MHz}, \mathrm{CDCl}_{3}\right) \delta 1.03(\mathrm{t}, J=7.5 \mathrm{~Hz}, 3 \mathrm{H}), 1.50-1.80(\mathrm{~m}, 6 \mathrm{H})$, $2.08(\mathrm{~s}, 3 \mathrm{H}), 2.15-2.19(\mathrm{~m}, 1 \mathrm{H}), 2.40(\mathrm{t}, J=2.5 \mathrm{~Hz}, 1 \mathrm{H}), 3.67-3.72(\mathrm{~m}, 2 \mathrm{H}), 4.24-4.35(\mathrm{~m}, 2 \mathrm{H}), 4.99(\mathrm{~s}, 1 \mathrm{H})$, $5.11(\mathrm{~s}, 1 \mathrm{H}), 5.23(\mathrm{~s}, 1 \mathrm{H}) ;{ }^{13} \mathrm{C}$ NMR $\left(125 \mathrm{MHz}, \mathrm{CDCl}_{3}\right) \delta 12.0,18.2,21.3,24.5,25.9,29.0,49.4,62.5,73.4$, $76.7,80.4,99.5,112.5,146.4,169.7$; IR (neat) $\mathrm{cm}^{-1} 3284 \mathrm{~s}, 2969 \mathrm{~s}, 2945 \mathrm{~s}, 2875 \mathrm{~s}, 1750 \mathrm{~s}$; mass spectrum (LCMS) for $\mathrm{C}_{15} \mathrm{H}_{22} \mathrm{O}_{4}$ : m/e (relative intensity): 211 (20) (M-O-propargyl) ${ }^{+}, 151$ (100). 


\section{Pauson-Khand Cycloadducts.}

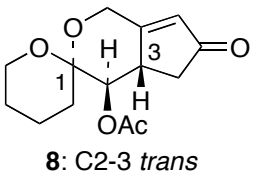

$\mathrm{R}_{f}=0.18$ [30\% EtOAc in hexanes]; ${ }^{1} \mathrm{H}$ NMR (500 MHz, $\left.\mathrm{CDCl}_{3}\right) \delta$ 1.49-1.65 (m, 5H), 1.80-1.92 (m, $\left.1 \mathrm{H}\right), 2.17$ (s, 3H), $2.25(\mathrm{dd}, J=3.0$ and $18.5 \mathrm{~Hz}, 1 \mathrm{H}), 2.49(\mathrm{dd}, J=6.0$ and $18.5 \mathrm{~Hz}, 1 \mathrm{H}), 3.38-3.41(\mathrm{~m}, 1 \mathrm{H}), 3.76-3.85(\mathrm{~m}$, $2 \mathrm{H}), 4.35-4.43(\mathrm{~m}, 2 \mathrm{H}), 4.72(\mathrm{~d}, J=11.0 \mathrm{~Hz}, 1 \mathrm{H}), 6.01(\mathrm{~s}, 1 \mathrm{H}) ;{ }^{13} \mathrm{C} \mathrm{NMR}\left(125 \mathrm{MHz}, \mathrm{CDCl}_{3}\right) \delta 18.0,21.0,24.6$, $29.8,39.1,40.3,59.1,62.0,77.5,96.3,128.4,170.5,172.6,206.8$; IR (neat) $\mathrm{cm}^{-1} 2944 \mathrm{~s}, 1740 \mathrm{~s}, 1714 \mathrm{~s}$; mass spectrum (LCMS) for $\mathrm{C}_{14} \mathrm{H}_{18} \mathrm{O}_{5}: \mathrm{m} / \mathrm{e}$ (relative intensity): 207 (100) (M-OAc) ${ }^{+}$.

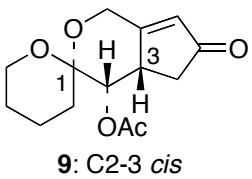

$\mathrm{R}_{f}=0.26$ [30\% EtOAc in hexanes]; ${ }^{1} \mathrm{H}$ NMR $\left(500 \mathrm{MHz}, \mathrm{CDCl}_{3}\right) \delta 1.38-1.43(\mathrm{~m}, 1 \mathrm{H}), 1.59-1.81(\mathrm{~m}, 5 \mathrm{H}), 1.96-$ $2.00(\mathrm{~m}, 1 \mathrm{H}), 2.06(\mathrm{~s}, 3 \mathrm{H}), 2.41(\mathrm{dd}, J=7.0$ and $18.5 \mathrm{~Hz}, 1 \mathrm{H}), 3.60(\mathrm{brs}, 1 \mathrm{H}), 3.73-3.77(\mathrm{~m}, 2 \mathrm{H}), 4.44-4.51(\mathrm{~m}$, $2 \mathrm{H}), 5.11(\mathrm{~d}, J=4.0 \mathrm{~Hz}, 1 \mathrm{H}), 6.00(\mathrm{~s}, 1 \mathrm{H}) ;{ }^{13} \mathrm{C} \mathrm{NMR}\left(125 \mathrm{MHz}, \mathrm{CDCl}_{3}\right) \delta 18.0,20.7,24.7,30.9,36.1,38.2$, 59.8, 61.6, 73.0, 95.7, 128.6, 170.5, 171.6, 207.1; IR (neat) $\mathrm{cm}^{-1} 2945 \mathrm{~s}, 2872 \mathrm{~m}, 1741 \mathrm{~s}, 1715 \mathrm{~s}, 1638 \mathrm{~m}$; mass spectrum (LCMS) for $\mathrm{C}_{14} \mathrm{H}_{18} \mathrm{O}_{5}: \mathrm{m} / \mathrm{e}$ (relative intensity): $267(100)(\mathrm{M}+\mathrm{H})^{+}, 207$ (82).

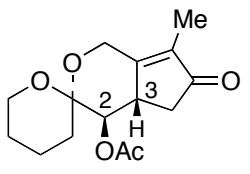

14

$\mathrm{R}_{f}=0.22\left[30 \%\right.$ EtOAc in hexanes]; ${ }^{1} \mathrm{H}$ NMR $\left(500 \mathrm{MHz}, \mathrm{CDCl}_{3}\right) \delta 1.45-1.70(\mathrm{~m}, 5 \mathrm{H}), 1.72(\mathrm{~s}, 3 \mathrm{H}), 1.79-1.86$ (m, 1H), $2.15(\mathrm{~s}, 3 \mathrm{H}), 2.14-2.20(\mathrm{~m}, 1 \mathrm{H}), 2.46(\mathrm{dd}, J=6.5$ and $19.0 \mathrm{~Hz}, 1 \mathrm{H}), 3.27-3.30(\mathrm{~m}, 1 \mathrm{H}), 3.74-3.85(\mathrm{~m}$, $2 \mathrm{H}), 4.30(\mathrm{~d}, J=13.5 \mathrm{~Hz}, 1 \mathrm{H}), 4.45(\mathrm{~d}, J=13.5 \mathrm{~Hz}, 1 \mathrm{H}), 4.64(\mathrm{~d}, J=11.0 \mathrm{~Hz}, 1 \mathrm{H}) ;{ }^{13} \mathrm{C} \mathrm{NMR}(125 \mathrm{MHz}$, $\left.\mathrm{CDCl}_{3}\right) \delta$ 7.9, 18.0, 21.0, 24.6, 29.9, 38.0, 38.6, 57.7, 61.9, 77.7, 96.5, 135.6, 163.9, 170.5, 207.1; IR (neat) $\mathrm{cm}^{-1}$ 2942s, 2875m, 1740s, 1704s, 1666s; mass spectrum (LCMS) for $\mathrm{C}_{15} \mathrm{H}_{20} \mathrm{O}_{5}: \mathrm{m} / \mathrm{e}$ (relative intensity): 221 (100) $(\mathrm{M}-\mathrm{OAc})^{+}$.

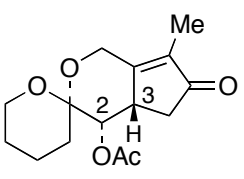

25

$\mathrm{R}_{f}=0.33\left[30 \%\right.$ EtOAc in hexanes]; ${ }^{1} \mathrm{H}$ NMR $\left(500 \mathrm{MHz}, \mathrm{CDCl}_{3}\right) \delta 1.34-1.41(\mathrm{~m}, 1 \mathrm{H}), 1.57-1.79(\mathrm{~m}, 5 \mathrm{H}), 1.77$ (s, 3H), $1.94(\mathrm{dd}, J=3.0$ and $18.5 \mathrm{~Hz}, 1 \mathrm{H}), 2.03(\mathrm{~s}, 3 \mathrm{H}), 2.39(\mathrm{dd}, J=6.5$ and $18.5 \mathrm{~Hz}, 1 \mathrm{H}), 3.49$ (brs, $1 \mathrm{H})$, 3.75-3.77 (m, 2H), $4.40(\mathrm{~d}, J=13.5 \mathrm{~Hz}, 1 \mathrm{H}), 4.55(\mathrm{~d}, J=13.5 \mathrm{~Hz}, 1 \mathrm{H}), 5.07(\mathrm{~d}, J=4.0 \mathrm{~Hz}, 1 \mathrm{H}) ;{ }^{13} \mathrm{C}$ NMR $(125$ 
$\left.\mathrm{MHz}, \mathrm{CDCl}_{3}\right) \delta 7.8,18.0,20.7,24.7,31.0,35.1,36.5,58.4,61.5,72.9,95.9,135.5,162.6,170.6,207.5 ;$ IR (neat) $\mathrm{cm}^{-1} 2944 \mathrm{~s}, 2874 \mathrm{~m}, 1744 \mathrm{~s}, 1706 \mathrm{~s}, 1668 \mathrm{~s}$; mass spectrum (LCMS) for $\mathrm{C}_{15} \mathrm{H}_{20} \mathrm{O}_{5}: \mathrm{m} / \mathrm{e}$ (relative intensity): $281(100)(\mathrm{M}+\mathrm{H})^{+}, 221(100)$.

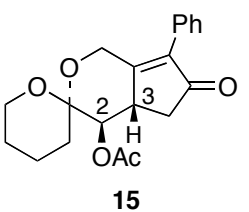

$\mathrm{R}_{f}=0.28$ [30\% EtOAc in hexanes]; ${ }^{1} \mathrm{H}$ NMR $\left(500 \mathrm{MHz}, \mathrm{CDCl}_{3}\right) \delta$ 1.47-1.67 (m, 5H), 1.78-1.84 (m, $\left.1 \mathrm{H}\right), 2.18$ (s, 3H), $2.39(\mathrm{dd}, J=3.0$ and $18.5 \mathrm{~Hz}, 1 \mathrm{H}), 2.64(\mathrm{dd}, J=6.0$ and $18.5 \mathrm{~Hz}, 1 \mathrm{H}), 3.50(\mathrm{ddd}, J=1.5,3.5$ and 8.0 $\mathrm{Hz}, 1 \mathrm{H}), 3.79$ (ddd, $J=2.5,11.5$ and $13.5 \mathrm{~Hz}, 1 \mathrm{H}), 3.87-3.90(\mathrm{~m}, 1 \mathrm{H}), 4.47-4.59(\mathrm{~m}, 2 \mathrm{H}), 4.78(\mathrm{~d}, J=10.5 \mathrm{~Hz}$, 1H), 7.23-7.26 (m, 2H), 7.33-7.41 (m, 3H); $\left.{ }^{13} \mathrm{C} \mathrm{NMR} \mathrm{(125} \mathrm{MHz,} \mathrm{CDCl}_{3}\right) \delta 18.0,21.0,24.6,29.9,38.5,39.0$, 77.6, 96.4, 128.4, 129.0, 130.0, 139.2, 165.8, 170.6, 204.7; IR (neat) $\mathrm{cm}^{-1} 2946 \mathrm{~s}, 2873 \mathrm{~m}, 1737 \mathrm{~s}, 1708 \mathrm{~s}$; mass spectrum (LCMS) for $\mathrm{C}_{20} \mathrm{H}_{22} \mathrm{O}_{5}: \mathrm{m} / \mathrm{e}$ (relative intensity): 283 (100) (M-OAc) ${ }^{+}$.

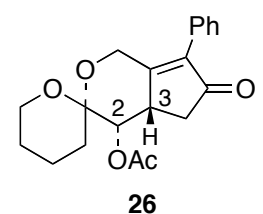

$\mathrm{R}_{f}=0.42\left[30 \%\right.$ EtOAc in hexanes]; ${ }^{1} \mathrm{H}$ NMR $\left(500 \mathrm{MHz}, \mathrm{CDCl}_{3}\right) \delta 1.38-1.56(\mathrm{~m}, 1 \mathrm{H}), 1.59-1.77(\mathrm{~m}, 5 \mathrm{H}), 2.07$ $(\mathrm{s}, 3 \mathrm{H}), 2.16(\mathrm{dd}, J=3.0$ and $18.5 \mathrm{~Hz}, 1 \mathrm{H}), 2.58(\mathrm{dd}, J=7.5$ and $19.5 \mathrm{~Hz}, 1 \mathrm{H}), 3.69$ (brs, $1 \mathrm{H}), 3.78-3.81(\mathrm{~m}$, $2 \mathrm{H}), 4.57-4.69(\mathrm{~m}, 2 \mathrm{H}), 5.15(\mathrm{~d}, J=3.5 \mathrm{~Hz}, 1 \mathrm{H}), 7.26-7.28(\mathrm{~m}, 2 \mathrm{H}), 7.32-7.42(\mathrm{~m}, 3 \mathrm{H}) ;{ }^{13} \mathrm{C} \mathrm{NMR}(125 \mathrm{MHz}$, $\left.\mathrm{CDCl}_{3}\right) \delta 18.1,20.7,24.8,30.9,36.0,36.4,58.9,61.6,73.0,95.7,128.3,129.0,130.1,139.3,164.8,170.6$, 205.0; IR (neat) $\mathrm{cm}^{-1} 2944 \mathrm{~s}, 2873 \mathrm{~m}, 1746 \mathrm{~s}, 1705 \mathrm{~s}$; mass spectrum (LCMS) for $\mathrm{C}_{20} \mathrm{H}_{22} \mathrm{O}_{5}$ : m/e (relative intensity): $431(95)(\mathrm{M}+\mathrm{H})^{+}, 283(100)$.

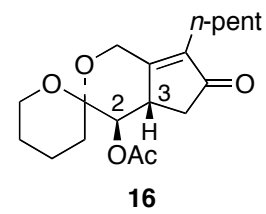

$\mathrm{R}_{f}=0.48\left[30 \%\right.$ EtOAc in hexanes]; ${ }^{1} \mathrm{H}$ NMR $\left(500 \mathrm{MHz}, \mathrm{CDCl}_{3}\right) \delta 0.87(\mathrm{t}, J=7.0 \mathrm{~Hz}, 3 \mathrm{H}), 1.20-1.87(\mathrm{~m}, 12 \mathrm{H})$, 2.10-2.24 (m, 3H), $2.16(\mathrm{~s}, 3 \mathrm{H}), 2.47(\mathrm{dd}, J=6.5$ and $15.5 \mathrm{~Hz}, 1 \mathrm{H}), 3.29-3.32(\mathrm{~m}, 1 \mathrm{H}), 3.76-3.87(\mathrm{~m}, 2 \mathrm{H}), 4.31$ $(\mathrm{d}, J=14.5 \mathrm{~Hz}, 1 \mathrm{H}), 4.47(\mathrm{~d}, J=14.5 \mathrm{~Hz}, 1 \mathrm{H}), 4.65(\mathrm{~d}, J=11.5 \mathrm{~Hz}, 1 \mathrm{H}) ;{ }^{13} \mathrm{C} \mathrm{NMR}\left(125 \mathrm{MHz}, \mathrm{CDCl}_{3}\right) \delta 13.9$, 18.0, 21.0, 22.3, 22.9, 24.6, 28.3, 29.9, 31.6, 38.2, 38.5, 57.6, 61.9, 77.8, 96.4, 140.0, 164.1, 170.5, 206.8; IR (neat) $\mathrm{cm}^{-1} 2933 \mathrm{~s}, 2868 \mathrm{~m}, 1745 \mathrm{~s}, 1705 \mathrm{~s}$; mass spectrum (LCMS) for $\mathrm{C}_{19} \mathrm{H}_{28} \mathrm{O}_{5}: \mathrm{m} / \mathrm{e}$ (relative intensity): 337 (47) $(\mathrm{M}+\mathrm{H})^{+}, 277$ (100). 


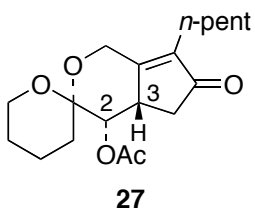

$\mathrm{R}_{f}=0.64$ [30\% EtOAc in hexanes]; ${ }^{1} \mathrm{H} \mathrm{NMR}\left(500 \mathrm{MHz}, \mathrm{CDCl}_{3}\right) \delta 0.87(\mathrm{t}, J=7.0 \mathrm{~Hz}, 3 \mathrm{H}), 1.20-1.81(\mathrm{~m}, 12 \mathrm{H})$, $1.95(\mathrm{dd}, J=1.5$ and $19.0 \mathrm{~Hz}, 1 \mathrm{H}), 2.03(\mathrm{~s}, 3 \mathrm{H}), 2.12-2.17(\mathrm{~m}, 1 \mathrm{H}), 2.22-2.27(\mathrm{~m}, 1 \mathrm{H}), 3.40(\mathrm{dd}, J=7.0$ and $18.5 \mathrm{~Hz}, 1 \mathrm{H}), 3.50-3.52(\mathrm{~m}, 1 \mathrm{H}), 3.77-3.80(\mathrm{~m}, 1 \mathrm{H}), 4.41(\mathrm{~d}, J=13.5 \mathrm{~Hz}, 1 \mathrm{H}), 4.55(\mathrm{~d}, J=13.5 \mathrm{~Hz}, 1 \mathrm{H}), 5.09$ $(\mathrm{d}, J=4.0 \mathrm{~Hz}, 1 \mathrm{H}) ;{ }^{13} \mathrm{C} \mathrm{NMR}\left(125 \mathrm{MHz}, \mathrm{CDCl}_{3}\right) \delta$ 14.0, 18.1, 20.6, 22.4, 22.8, 24.8, 28.2, 31.0, 31.4, 35.3, $36.3,58.4,61.4,73.0,95.8,140.0,162.9,170.5,207.2$; IR (neat) $\mathrm{cm}^{-1} 2934 \mathrm{~s}, 2868 \mathrm{~m}, 1747 \mathrm{~s}, 1708 \mathrm{~s}$; mass spectrum (LCMS) for $\mathrm{C}_{19} \mathrm{H}_{28} \mathrm{O}_{5}: \mathrm{m} / \mathrm{e}$ (relative intensity): 337 (47) (M+H) ${ }^{+}, 277$ (100).

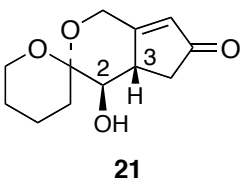

$\mathrm{R}_{f}=0.38$ [50\% EtOAc in hexanes]; ${ }^{1} \mathrm{H}$ NMR $\left(500 \mathrm{MHz}, \mathrm{CDCl}_{3}\right) \delta 1.45-1.48(\mathrm{~m}, 1 \mathrm{H}), 1.55-1.67(\mathrm{~m}, 3 \mathrm{H}), 1.75-$ $1.87(\mathrm{~m}, 1 \mathrm{H}), 2.00-2.09(\mathrm{~m}, 1 \mathrm{H}), 2.22-2.34(\mathrm{~m}, 2 \mathrm{H}), 2.63-2.68(\mathrm{~m}, 1 \mathrm{H}), 3.07-3.11(\mathrm{~m}, 1 \mathrm{H}), 3.75-3.82(\mathrm{~m}, 2 \mathrm{H})$, 4.30-4.39 (m, 2H), 5.96 (s, 1H); ${ }^{13} \mathrm{C}$ NMR (125 MHz, $\left.\mathrm{CDCl}_{3}\right)$ ઈ 18.1, 24.7, 29.5, 39.8, 43.4, 59.0, 61.8, 78.9, 97.0, 127.8, 173.7, 208.1; IR (neat) $\mathrm{cm}^{-1} 3427 \mathrm{~s}, 2941 \mathrm{~s}, 2870 \mathrm{~m}, 1705 \mathrm{~s}, 1633 \mathrm{~s}$; mass spectrum (LCMS) for $\mathrm{C}_{12} \mathrm{H}_{16} \mathrm{O}_{4}: \mathrm{m} / \mathrm{e}$ (relative intensity): $225(100)(\mathrm{M}+\mathrm{H})^{+}, 207(60)$.

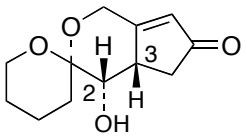

37-cis

$\mathrm{R}_{f}=0.33$ [50\% EtOAc in hexanes]; ${ }^{1} \mathrm{H}$ NMR $\left(500 \mathrm{MHz}, \mathrm{CDCl}_{3}\right) \delta 1.40-1.46(\mathrm{~m}, 1 \mathrm{H}), 1.56-1.82(\mathrm{~m}, 4 \mathrm{H}), 1.95-$ $2.02(\mathrm{~m}, 2 \mathrm{H}), 2.33(\mathrm{dd}, J=7.0$ and $14.0 \mathrm{~Hz}, 1 \mathrm{H}), 2.50(\mathrm{dd}, J=3.0$ and $14.0 \mathrm{~Hz}, 1 \mathrm{H}), 3.50(\mathrm{brs}, 1 \mathrm{H}), 3.58(\mathrm{dd}, J$ $=3.5$ and $10.5 \mathrm{~Hz}, 1 \mathrm{H}), 3.71-3.78(\mathrm{~m}, 2 \mathrm{H}), 4.40(\mathrm{~s}, 2 \mathrm{H}), 6.00(\mathrm{~s}, 1 \mathrm{H}) ;{ }^{13} \mathrm{C} \mathrm{NMR}\left(125 \mathrm{MHz}, \mathrm{CDCl}_{3}\right) \delta 18.2$, 24.8, 31.1, 36.2, 40.4, 59.8, 61.7, 73.2, 97.0, 129.5, 172.2, 208.6; IR (neat) cm $\mathrm{cm}^{-1} 3426 \mathrm{~s}, 2941 \mathrm{~s}, 2871 \mathrm{~m}, 1707 \mathrm{~s}$; mass spectrum (LCMS) for $\mathrm{C}_{12} \mathrm{H}_{16} \mathrm{O}_{4}: \mathrm{m} / \mathrm{e}$ (relative intensity): 225 (100) $(\mathrm{M}+\mathrm{H})^{+}, 207$ (52).

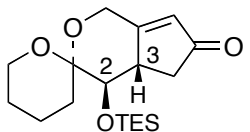

22

$\mathrm{R}_{f}=0.53\left[30 \%\right.$ EtOAc in hexanes]; ${ }^{1} \mathrm{H}$ NMR $\left(500 \mathrm{MHz}, \mathrm{CDCl}_{3}\right) \delta 0.67(\mathrm{q}, J=8.0 \mathrm{~Hz}, 6 \mathrm{H}), 0.99(\mathrm{t}, J=8.0 \mathrm{~Hz}$, 9H), 1.39-1.41 (m, 1H), 1.53-1.69 (m, 3H), 1.82-1.87 (m, 1H), $1.96(\mathrm{ddd}, J=4.5,13.0$ and $17.5 \mathrm{~Hz}, 1 \mathrm{H}) 2.18$ $(\mathrm{dd}, J=2.5$ and $18.5 \mathrm{~Hz}, 1 \mathrm{H}), 2.61(\mathrm{dd}, J=5.0$ and $18.5 \mathrm{~Hz}, 1 \mathrm{H}), 3.25-3.39(\mathrm{~m}, 2 \mathrm{H}), 3.68-3.74(\mathrm{~m}, 1 \mathrm{H}), 3.83-$ $3.86(\mathrm{~m}, 1 \mathrm{H}), 4.32(\mathrm{~s}, 2 \mathrm{H}), 5.92(\mathrm{~s}, 1 \mathrm{H}) ;{ }^{13} \mathrm{C} \mathrm{NMR}\left(125 \mathrm{MHz}, \mathrm{CDCl}_{3}\right) \delta$-5.5, -7.0, 18.4, 24.7, 29.8, 40.5, 43.0, 
59.0, 61.8, 79.9, 97.5, 127.5, 174.8, 207.8; IR (neat) $\mathrm{cm}^{-1} 2953 \mathrm{~s}, 2878 \mathrm{~s}, 1716 \mathrm{~s}$; mass spectrum (LCMS) for $\mathrm{C}_{18} \mathrm{H}_{30} \mathrm{O}_{4} \mathrm{Si}: \mathrm{m} / \mathrm{e}$ (relative intensity): $339(100)(\mathrm{M}+\mathrm{H})^{+}, 207$ (50).

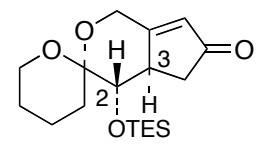

38-trans

$\mathrm{R}_{f}=0.27$ [20\% EtOAc in hexanes]; ${ }^{1} \mathrm{H}$ NMR $\left(500 \mathrm{MHz}, \mathrm{CDCl}_{3}\right) \delta$ 0.58-0.70 (m, 6H), 0.91-0.98 (m, 9H), 1.53$1.80(\mathrm{~m}, 5 \mathrm{H}), 1.90-1.94(\mathrm{~m}, 1 \mathrm{H}), 2.20(\mathrm{dd}, J=3.0$ and $18.5 \mathrm{~Hz}, 1 \mathrm{H}), 2.32-2.34(\mathrm{~m}, 1 \mathrm{H}), 2.64(\mathrm{dd}, J=6.0$ and $18.5 \mathrm{~Hz}, 1 \mathrm{H}), 2.95-2.98(\mathrm{~m}, 1 \mathrm{H}), 3.34(\mathrm{~d}, J=10.0 \mathrm{~Hz}, 1 \mathrm{H}), 3.68-3.76(\mathrm{~m}, 1 \mathrm{H}), 3.84-3.89(\mathrm{~m}, 1 \mathrm{H}), 4.36(\mathrm{~d}, J=$

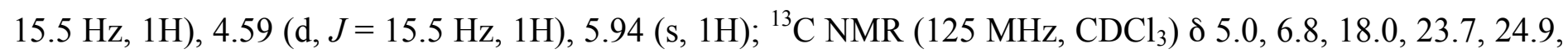
40.6, 44.7, 61.2, 62.1, 80.5, 99.9, 127.0, 175.3, 207.5; IR (neat) $\mathrm{cm}^{-1} 2952 \mathrm{~s}, 2880 \mathrm{~s}, 1715 \mathrm{~s}, 1635 \mathrm{w}$; mass spectrum (LCMS) for $\mathrm{C}_{18} \mathrm{H}_{30} \mathrm{O}_{4} \mathrm{Si}: \mathrm{m} / \mathrm{e}$ (relative intensity): 339 (100) $(\mathrm{M}+\mathrm{H})^{+}, 207$ (27).

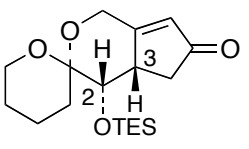

38-cis

$\mathrm{R}_{f}=0.28$ [20\% EtOAc in hexanes]; ${ }^{1} \mathrm{H} \mathrm{NMR}\left(500 \mathrm{MHz}, \mathrm{CDCl}_{3}\right) \delta$ 0.59-0.64 (m, 6H), $0.93(\mathrm{t}, J=8.0 \mathrm{~Hz}, 9 \mathrm{H})$, $1.31(\mathrm{ddd}, J=5.0,13.5$ and $18.5 \mathrm{~Hz}, 1 \mathrm{H}), 1.57-1.70(\mathrm{~m}, 3 \mathrm{H}), 1.79-1.98(\mathrm{~m}, 2 \mathrm{H}), 2.32-2.34(\mathrm{~m}, 2 \mathrm{H}), 3.50(\mathrm{brs}$, $1 \mathrm{H}), 3.74-3.78(\mathrm{~m}, 3 \mathrm{H}), 4.38(\mathrm{~d}, J=13.5 \mathrm{~Hz}, 1 \mathrm{H}), 4.47(\mathrm{~d}, J=13.5 \mathrm{~Hz}, 1 \mathrm{H}), 5.95(\mathrm{~s}, 1 \mathrm{H}) ;{ }^{13} \mathrm{C} \mathrm{NMR}(125 \mathrm{MHz}$ $\left.\mathrm{CDCl}_{3}\right) \delta$ 5.4, 6.9, 18.4, 25.0, 32.0, 36.5, 40.6, 59.9, 61.6, 74.8, 96.8, 127.9, 173.7, 208.2; IR (neat) $\mathrm{cm}^{-1} 2952 \mathrm{~s}$, 2880s, 1715s; mass spectrum (LCMS) for $\mathrm{C}_{18} \mathrm{H}_{30} \mathrm{O}_{4} \mathrm{Si}: \mathrm{m} / \mathrm{e}$ (relative intensity): $339(100)(\mathrm{M}+\mathrm{H})^{+}, 207(70)$.

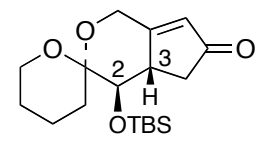

23

$\mathrm{R}_{f}=0.40\left[20 \%\right.$ EtOAc in hexanes]; ${ }^{1} \mathrm{H}$ NMR $\left(500 \mathrm{MHz}, \mathrm{CDCl}_{3}\right) \delta-0.11(\mathrm{~s}, 6 \mathrm{H}), 0.91(\mathrm{~s}, 9 \mathrm{H}), 1.37(\mathrm{~d}, J=10.0$ $\mathrm{Hz}, 1 \mathrm{H}), 1.53-1.67(\mathrm{~m}, 3 \mathrm{H}), 1.79-1.87(\mathrm{~m}, 1 \mathrm{H}), 1.99(\mathrm{ddd}, J=4.5,13.0$ and $13.0 \mathrm{~Hz}, 1 \mathrm{H}), 2.17-2.21(\mathrm{~m}, 1 \mathrm{H})$, 2.56-2.61 (m, 1H), $3.28(\mathrm{~s}, 2 \mathrm{H}), 3.68-3.73(\mathrm{~m}, 1 \mathrm{H}), 3.80-3.83(\mathrm{~m}, 1 \mathrm{H}), 4.32(\mathrm{~s}, 2 \mathrm{H}), 5.92(\mathrm{~s}, 1 \mathrm{H}) ;{ }^{13} \mathrm{C} \mathrm{NMR}$ $\left(125 \mathrm{MHz}, \mathrm{CDCl}_{3}\right) \delta$-3.8, -2.9, 18.4, 24.7, 26.1, 29.9, 41.0, 42.9, 59.0, 61.7, 80.2, 97.5, 127.3, 174.9, 207.8; IR (neat) $\mathrm{cm}^{-1} 2953 \mathrm{~s}, 2932 \mathrm{~s}, 2857 \mathrm{~s}, 1716 \mathrm{~s}$; mass spectrum (LCMS) for $\mathrm{C}_{18} \mathrm{H}_{30} \mathrm{O}_{4} \mathrm{Si}: \mathrm{m} / \mathrm{e}$ (relative intensity): 339 (100) $(\mathrm{M}+\mathrm{H})^{+}, 207$ (95). 


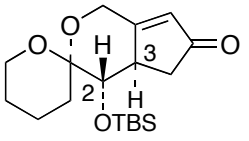

39-trans

$\mathrm{R}_{f}=0.40$ [30\% EtOAc in hexanes]; ${ }^{1} \mathrm{H}$ NMR $\left(500 \mathrm{MHz}, \mathrm{CDCl}_{3}\right) \delta 0.06(\mathrm{~s}, 3 \mathrm{H}), 0.12(\mathrm{~s}, 3 \mathrm{H}), 0.89$ (s, $\left.9 \mathrm{H}\right), 1.55-$ $1.94(\mathrm{~m}, 6 \mathrm{H}), 2.19(\mathrm{dd}, J=3.0$ and $19.0 \mathrm{~Hz}, 1 \mathrm{H}), 2.63(\mathrm{dd}, J=6.5$ and $19.0 \mathrm{~Hz}, 1 \mathrm{H}), 3.95-3.00(\mathrm{~m}, 1 \mathrm{H}), 3.31$ $(\mathrm{d}, J=10.0 \mathrm{~Hz}, 1 \mathrm{H}), 3.66-3.71(\mathrm{~m}, 1 \mathrm{H}), 3.86-3.89(\mathrm{~m}, 1 \mathrm{H}), 4.36(\mathrm{~d}, J=15.5 \mathrm{~Hz}, 1 \mathrm{H}), 4.59(\mathrm{~d}, J=15.0 \mathrm{~Hz}, 1 \mathrm{H})$, $5.94(\mathrm{~s}, 1 \mathrm{H}) ;{ }^{13} \mathrm{C}$ NMR $\left(125 \mathrm{MHz}, \mathrm{CDCl}_{3}\right) \delta$-4.9, -3.9, 17.9, 23.4, 24.9, 25.8, 25.9, 40.8, 44.6, 61.2, 62.0, 80.4, 100.1, 127.1, 175.2, 206.9; IR (neat) $\mathrm{cm}^{-1} 2953 \mathrm{~s}, 2934 \mathrm{~s}, 2859 \mathrm{~s}, 1717 \mathrm{~s}$; mass spectrum (LCMS) for $\mathrm{C}_{18} \mathrm{H}_{30} \mathrm{O}_{4} \mathrm{Si}_{\text {: }}$ m/e (relative intensity): $339(80)(\mathrm{M}+\mathrm{H})^{+}, 207(100)$.

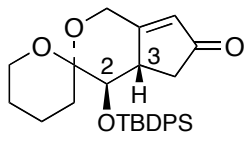

24

$\mathrm{R}_{f}=0.30\left[20 \%\right.$ EtOAc in hexanes]; ${ }^{1} \mathrm{H}$ NMR $\left(500 \mathrm{MHz}, \mathrm{CDCl}_{3}\right) \delta 1.03(\mathrm{~s}, 9 \mathrm{H}), 1.03-1.11(\mathrm{~m}, 1 \mathrm{H}), 1.52-1.58$ (m, 2H), 1.62-1.78 (m, 2H), $1.83(\mathrm{dd}, J=3.0$ and $19.0 \mathrm{~Hz}, 1 \mathrm{H}), 2.13(\mathrm{ddd}, J=5.0,13.0$ and $18.0 \mathrm{~Hz}, 1 \mathrm{H}), 2.26$ $(\mathrm{dd}, J=6.0$ and $18.5 \mathrm{~Hz}, 1 \mathrm{H}), 3.26(\mathrm{~d}, J=9.5 \mathrm{~Hz}, 1 \mathrm{H}), 3.34-3.37$ (m, 1H), 3.73 (ddd, $J=2.5$ and 13.0 and 13.0 $\mathrm{Hz}, 1 \mathrm{H}), 3.91(\mathrm{dd}, J=4.0$ and $10.5 \mathrm{~Hz}, 1 \mathrm{H}), 4.23(\mathrm{~d}, J=13.0 \mathrm{~Hz}, 1 \mathrm{H}), 4.30(\mathrm{~d}, J=13.0 \mathrm{~Hz}, 1 \mathrm{H}), 5.80(\mathrm{~s}, 1 \mathrm{H})$, 7.36-7.44 (m, 6H), 7.72-7.77 (m, 4H); ${ }^{13} \mathrm{C}$ NMR (125 MHz, $\left.\mathrm{CDCl}_{3}\right)$ ठ 18.4, 19.8, 24.5, 27.1, 29.8, 41.5, 43.2, $58.7,61.8,80.5,97.5,127.4,127.6,129.9,130.0,132.7,133.1,136.0,136.1,174.4,207.8 ;$ IR $($ neat $) \mathrm{cm}^{-1}$ 2935s, 2859s, 1713s; mass spectrum (LCMS) for $\mathrm{C}_{28} \mathrm{H}_{34} \mathrm{O}_{4} \mathrm{Si}: \mathrm{m} / \mathrm{e}$ (relative intensity): $463(100)(\mathrm{M}+\mathrm{H})^{+}$.

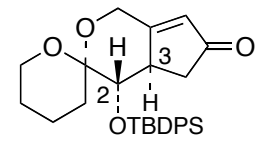

40-trans

$\mathrm{R}_{f}=0.36$ [30\% EtOAc in hexanes]; ${ }^{1} \mathrm{H}$ NMR $\left(500 \mathrm{MHz}, \mathrm{CDCl}_{3}\right) \delta 1.07(\mathrm{~s}, 9 \mathrm{H}), 1.56-1.60(\mathrm{~m}, 3 \mathrm{H}), 1.74-1.77$ (m, 2H), 1.98-2.04 (m, 1H), $2.13(\mathrm{dd}, J=6.0$ and $18.5 \mathrm{~Hz}, 1 \mathrm{H}), 3.03$ (brs, 1H), $3.46(\mathrm{~d}, J=9.0 \mathrm{~Hz}, 1 \mathrm{H}), 3.65$ $(\mathrm{dd}, J=5.0$ and $11.0 \mathrm{~Hz}, 1 \mathrm{H}), 3.81-3.83(\mathrm{~m}, 1 \mathrm{H}), 4.36(\mathrm{~d}, J=15.0 \mathrm{~Hz}, 1 \mathrm{H}), 4.53(\mathrm{~d}, J=15.0 \mathrm{~Hz}, 1 \mathrm{H}), 5.79(\mathrm{~s}$, 1H), 7.35-7.43 (m, 6H), 7.70-7.73 (m, 4H); $\left.{ }^{13} \mathrm{C} \mathrm{NMR} \mathrm{(125} \mathrm{MHz,} \mathrm{CDCl}_{3}\right) \delta$ 18.0, 19.7, 24.3, 24.8, 26.9, 41.3, $44.8,61.3,62.0,80.7,99.9,127.0,127.6,129.8,130.0,132.3,133.6,135.9,136.3,174.4,207.2 ;$ IR $($ neat $) \mathrm{cm}^{-1}$ $2957 \mathrm{~s}, 2942 \mathrm{~s}, 2857 \mathrm{~s}, 1714 \mathrm{~s}$; mass spectrum (LCMS) for $\mathrm{C}_{28} \mathrm{H}_{34} \mathrm{O}_{4} \mathrm{Si}: \mathrm{m} / \mathrm{e}$ (relative intensity): $463(80)(\mathrm{M}+\mathrm{H})^{+}$, $385(100)$.

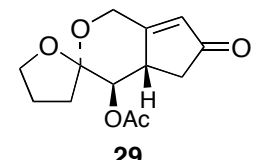


$\mathrm{R}_{f}=0.20$ [30\% EtOAc in hexanes]; ${ }^{1} \mathrm{H}$ NMR $\left(500 \mathrm{MHz}, \mathrm{CDCl}_{3}\right) \delta 1.81-1.94(\mathrm{~m}, 3 \mathrm{H}), 2.06-2.12(\mathrm{~m}, 1 \mathrm{H}), 2.14$ (s, 3H), $2.28(\mathrm{dd}, J=3.0$ and $19.0 \mathrm{~Hz}, 1 \mathrm{H}), 2.52(\mathrm{dd}, J=6.5$ and $19.0 \mathrm{~Hz}, 1 \mathrm{H}), 3.37-3.40(\mathrm{~m}, 1 \mathrm{H}), 4.01-4.06(\mathrm{~m}$, 2H), $4.38(\mathrm{~d}, J=13.5 \mathrm{~Hz}, 1 \mathrm{H}), 4.54(\mathrm{~d}, J=13.5 \mathrm{~Hz}, 1 \mathrm{H}), 4.96(\mathrm{~d}, J=11.0 \mathrm{~Hz}, 1 \mathrm{H}), 6.01(\mathrm{~s}, 1 \mathrm{H}) ;{ }^{13} \mathrm{C} \mathrm{NMR}(125$ $\left.\mathrm{MHz}, \mathrm{CDCl}_{3}\right) \delta$ 20.9, 24.0, 33.2, 39.3, 42.0, 60.0, 68.6, 74.8, 105.8, 128.4, 170.5, 172.4, 206.7; IR (neat) $\mathrm{cm}^{-1}$ $22959 \mathrm{~m}, 2890 \mathrm{~m}, 111748 \mathrm{~s}, 1716 \mathrm{~s}$; mass spectrum (LCMS) for $\mathrm{C}_{13} \mathrm{H}_{16} \mathrm{O}_{5}: \mathrm{m} / \mathrm{e}$ (relative intensity): 193 (M-OAc) ${ }^{+}$ (100).

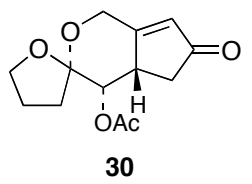

$\mathrm{R}_{f}=0.24\left[30 \%\right.$ EtOAc in hexanes]; ${ }^{1} \mathrm{H}$ NMR $\left(500 \mathrm{MHz}, \mathrm{CDCl}_{3}\right) \delta 1.81-2.14(\mathrm{~m}, 5 \mathrm{H}), 2.11(\mathrm{~s}, 3 \mathrm{H}), 2.45(\mathrm{dd}, J$ $=7.0$ and $19.0 \mathrm{~Hz}, 1 \mathrm{H}), 3.57-3.58(\mathrm{~m}, 1 \mathrm{H}), 4.00-4.11(\mathrm{~m}, 2 \mathrm{H}), 4.48(\mathrm{~d}, J=13.5 \mathrm{~Hz}, 1 \mathrm{H}), 4.63(\mathrm{~d}, J=13.5 \mathrm{~Hz}$, $1 \mathrm{H}), 5.18(\mathrm{~d}, J=4.0 \mathrm{~Hz}, 1 \mathrm{H}), 6.02(\mathrm{~s}, 1 \mathrm{H}) ;{ }^{13} \mathrm{C}$ NMR $\left(125 \mathrm{MHz}, \mathrm{CDCl}_{3}\right) \delta$ 20.7, 23.7, 35.2, 36.2, 39.5, 60.6, 69.0, 72.5, 105.6, 128.5, 170.6, 171.4, 207.0; IR (neat) $\mathrm{cm}^{-1} 2975 \mathrm{~m}, 2893 \mathrm{~m}, 1739 \mathrm{~s}, 1716 \mathrm{~s}$; mass spectrum (LCMS) for $\mathrm{C}_{13} \mathrm{H}_{16} \mathrm{O}_{5}: \mathrm{m} / \mathrm{e}$ (relative intensity): 193 (M-OAc) ${ }^{+}(100)$.

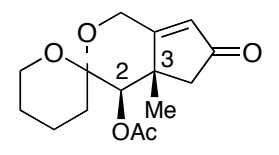

33

$\mathrm{R}_{f}=0.29$ [50\% EtOAc in hexanes]; ${ }^{1} \mathrm{H}$ NMR $\left(500 \mathrm{MHz}, \mathrm{CDCl}_{3}\right) \delta 1.46(\mathrm{~s}, 3 \mathrm{H}), 1.47(\mathrm{~m}, 1 \mathrm{H}), 1.54(\mathrm{~m}, 2 \mathrm{H})$, $1.62(\mathrm{~m}, 2 \mathrm{H}), 1.83(\mathrm{~m}, 1 \mathrm{H}), 2.15(\mathrm{~d}, J=19.0 \mathrm{~Hz}, 1 \mathrm{H}), 2.22(\mathrm{~s}, 3 \mathrm{H}), 2.50(\mathrm{~d}, J=19.0 \mathrm{~Hz}, 1 \mathrm{H}), 3.77$ (dt, $J=2.5$, $11.5 \mathrm{~Hz}, 1 \mathrm{H}), 3.84(\mathrm{~m}, 1 \mathrm{H}), 4.34(\mathrm{~d}, J=13.0 \mathrm{~Hz}, 1 \mathrm{H}), 4.47$ (d, $J=13.0 \mathrm{~Hz}, 1 \mathrm{H}), 4.79(\mathrm{~s}, 1 \mathrm{H}), 5.96(\mathrm{~s}, 1 \mathrm{H}) ;{ }^{13} \mathrm{C}$ NMR $\left(75 \mathrm{MHz}, \mathrm{CDCl}_{3}\right) \delta$ 17.9, 20.9, 21.7, 24.2, 30.7, 46.3, 51.1, 56.4, 61.4, 78.4, 97.3, 127.8, 170.4, 176.4, 206.4; IR (film) $\mathrm{cm}^{-1} 2943 \mathrm{~m}, 1717 \mathrm{~s}$; mass spectrum (APCI) for $\mathrm{C}_{15} \mathrm{H}_{20} \mathrm{O}_{5}: \mathrm{m} / \mathrm{e}$ (\%relative intensity) 281.1 $(\mathrm{M}+\mathrm{H})^{+}(20), 221.1(100)$.

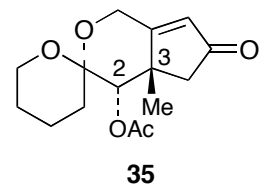

$\mathrm{R}_{f}=0.35\left[50 \%\right.$ EtOAc in hexanes]; ${ }^{1} \mathrm{H}$ NMR $\left(500 \mathrm{MHz}, \mathrm{CDCl}_{3}\right) \delta 1.37(\mathrm{~m}, 1 \mathrm{H}), 1.59(\mathrm{~s}, 3 \mathrm{H}), 1.61(\mathrm{~m}, 4 \mathrm{H})$, $1.77(\mathrm{~m}, 1 \mathrm{H}), 2.05(\mathrm{~d}, J=18.5 \mathrm{~Hz}, 1 \mathrm{H}), 2.08(\mathrm{~s}, 3 \mathrm{H}), 2.27$ (d, $J=18.0 \mathrm{~Hz}, 1 \mathrm{H}), 3.77(\mathrm{~m}, 2 \mathrm{H}), 4.40$ (d, $J=12.5$ $\mathrm{Hz}, 1 \mathrm{H}), 4.53(\mathrm{~d}, J=12.5 \mathrm{~Hz}, 1 \mathrm{H}), 4.93(\mathrm{~s}, 1 \mathrm{H}), 5.80(\mathrm{~s}, 1 \mathrm{H}) ;{ }^{13} \mathrm{C} \mathrm{NMR}\left(75 \mathrm{MHz}, \mathrm{CDCl}_{3}\right) \delta$ 18.0, 20.6, 24.3, 25.8, 31.0, 44.7, 47.4, 57.0, 60.9, 76.3, 97.3, 128.1, 170.1, 175.2, 206.5; IR (film) $\mathrm{cm}^{-1} 2942 \mathrm{~m}, 1746 \mathrm{~s}, 1717 \mathrm{~s}$; mass spectrum (APCI) for $\mathrm{C}_{15} \mathrm{H}_{20} \mathrm{O}_{5}: \mathrm{m} / \mathrm{e}\left(\%\right.$ relative intensity) $281.1(\mathrm{M}+\mathrm{H})^{+}(20), 221.1$ (M-OAc) ${ }^{+}(100)$. 


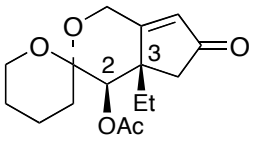

34

$\mathrm{R}_{f}=0.22\left[20 \%\right.$ EtOAc in hexanes]; ${ }^{1} \mathrm{H} \mathrm{NMR}\left(500 \mathrm{MHz}, \mathrm{CDCl}_{3}\right) \delta 0.63(\mathrm{t}, J=7.5 \mathrm{~Hz}, 3 \mathrm{H}), 1.48-1.84(\mathrm{~m}, 7 \mathrm{H})$, $2.19(\mathrm{~s}, 3 \mathrm{H}), 2.25-2.49(\mathrm{~m}, 3 \mathrm{H}), 3.74-3.81(\mathrm{~m}, 2 \mathrm{H}), 4.27-4.34(\mathrm{~m}, 2 \mathrm{H}), 4.83(\mathrm{~s}, 1 \mathrm{H}), 6.05(\mathrm{~s}, 1 \mathrm{H}) ;{ }^{13} \mathrm{C} \mathrm{NMR}$ $\left(125 \mathrm{MHz}, \mathrm{CDCl}_{3}\right) \delta$ 7.9, 18.1, 21.0, 23.7, 24.3, 31.2, 47.7, 50.4, 56.5, 61.4, 79.7, 97.6, 129.7, 170.4, 173.6, 206.5; IR (neat) cm $\mathrm{cm}^{-1} 2965 \mathrm{~s}, 2943 \mathrm{~s}, 2876 \mathrm{~m}, 1739 \mathrm{~s}, 1717 \mathrm{~s}$; mass spectrum (LCMS) for $\mathrm{C}_{16} \mathrm{H}_{22} \mathrm{O}_{5}$ : m/e (relative intensity): 295 (100) $(\mathrm{M}+\mathrm{H})^{+}, 235$ (62).

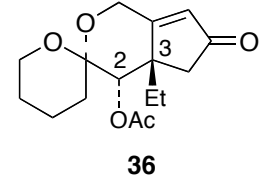

$\mathrm{R}_{f}=0.23\left[20 \%\right.$ EtOAc in hexanes]; ${ }^{1} \mathrm{H} \mathrm{NMR}\left(500 \mathrm{MHz}, \mathrm{CDCl}_{3}\right) \delta 0.88(\mathrm{t}, J=7.0 \mathrm{~Hz}, 3 \mathrm{H}), 1.33-1.92(\mathrm{~m}, 7 \mathrm{H})$, $2.05(\mathrm{~s}, 3 \mathrm{H}), 2.11(\mathrm{~s}, 2 \mathrm{H}), 2.11-2.20(\mathrm{~m}, 1 \mathrm{H}), 3.74(\mathrm{~d}, J=7.5 \mathrm{~Hz}, 1 \mathrm{H}), 3.36(\mathrm{~d}, J=12.5 \mathrm{~Hz}, 1 \mathrm{H}), 4.46(\mathrm{~d}, J=$ $12.5 \mathrm{~Hz}, 1 \mathrm{H}), 5.02$ (s, 1H), 6.01 (s, 1H); $\left.{ }^{13} \mathrm{C} \mathrm{NMR} \mathrm{(125} \mathrm{MHz,} \mathrm{CDCl}_{3}\right) \delta$ 9.3, 18.2, 20.7, 24.4, 29.5, 31.2, 44.2, 49.0, 57.2, 60.9, 74.6, 97.6, 129.0, 170.1, 174.5, 206.7; IR (neat) $\mathrm{cm}^{-1} 2963 \mathrm{~s}, 2945 \mathrm{~s}, 2875 \mathrm{~m}, 1737 \mathrm{~s}, 1715 \mathrm{~s} ;$ mass spectrum (LCMS) for $\mathrm{C}_{16} \mathrm{H}_{22} \mathrm{O}_{5}: \mathrm{m} / \mathrm{e}$ (relative intensity): $295(100)(\mathrm{M}+\mathrm{H})^{+}, 235$ (60). 